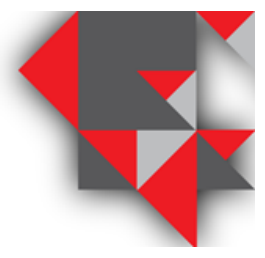

\title{
Factores que inciden en la cultura financiera de los estudiantes de maestría de la Facultad de Contaduría Pública y Administración de la UANL
}

\author{
Gustavo Adolfo Treviño Enríquez ${ }^{1}$ y Klender Aimer Cortez Alejandro ${ }^{2}$ \\ ${ }^{1}$ Universidad Autónoma de Nuevo León, gtrevino16@gmail.com, Ciudad Universitaria, 8113404430 \\ ${ }^{2}$ Universidad Autónoma de Nuevo León, klender.cortezal@uanl.edu.mx, Ciudad Universitaria, 8113404430
}

Información del artículo revisado por pares

Fecha de aceptación: junio-2021

Fecha de publicación en línea: diciembre-2021

DOI: https://doi.org/10.29105/vtga7.1-110

\section{Resumen}

El presente artículo estudia los factores que influyen en la cultura financiera de los estudiantes de maestría de la Facultad de Contaduría Pública y Administración de la Universidad Autónoma de Nuevo León. Los factores analizados en el estudio son el género, la educación financiera, la bancarización y la regulación financiera. Para la recolección de datos se realizó un instrumento en forma de encuesta y se aplicó a estudiantes de maestría en esta institución. Para el análisis de los datos se utiliza la técnica de análisis factorial confirmatorio y posteriormente se aplica un modelo probit. Con los resultados obtenidos de la reducción de factores se descarta la incidencia de la regulación financiera y se concluye además que la educación financiera tiene una afectación importante en la cultura financiera de los estudiantes de maestría de la muestra

Palabras clave: bancarización y regulación, cultura financiera, educación financiera, finanzas personales

\section{INTRODUCCIÓN}

En México existen tres problemas relacionados con las finanzas personales: 1) ingresos insuficientes, 2) acceso al sistema financiero y 3) cultura financiera. La presente investigación se centra en estudiar los factores de género, educación financiera, bancarización y la regulación vigente como incidentes en la problemática en la cultura
Abstract

This article studies the factors that influence the financial culture of master's students at the School of Public Accounting and Administration of the Autonomous University of Nuevo Leon. The factors such as gender, financial education, banking and financial regulation are analyzed. For data collection, a survey was developed and applied to the master's students of this institution. For the analysis of the data, the confirmatory factor analysis technique is used and subsequently a probit model is applied. With the results obtained from the reduction of factors, the incidence of financial regulation is ruled out and it is also concluded that financial education has a significant impact on the financial culture of the master's students of the sample.

Keywords: banking and regulation, financial culture, financial education, personal finances

JEL: C35, C38, G50.

financiera. La falta de cultura financiera deriva en muchos problemas financieros tal y como lo establecen diversos autores en publicaciones y artículos relacionados al tema. En la medida en que los usuarios del sistema financiero cuenten con las herramientas adecuadas podrán tener cultura financiera y esto reduciría en alguna medida sus problemas financieros. 
Amezcua et al. (2014) señalan que el bajo nivel de cultura financiera en México es debido principalmente al nivel de educación financiera de la población, lo que conlleva a que se utilicen poco los productos y servicios financieros que existen, además a malos hábitos al momento de adquirirlos. Además, en el desconocimiento de sus derechos y obligaciones y la falta de planeación financiera, lo que incide negativamente en su patrimonio y calidad de vida. A su vez, esta problemática no ayuda a que la competitividad de las instituciones financieras $y$ por ende al desarrollo económico del país.

Por su parte, Polaina et al. (2016) realizan un estudio en países Latinoamericanos para identificar las políticas bancarias y programas formativos que impulsan la cultura financiera. En sus hallazgos encontraron aspectos relevantes que influyen en la cultura financiera, como la formación financiera, los indicadores macroeconómicos y las políticas bancarias, concluyendo que el nivel educativo es el eje principal, ya que incide de forma directa en la cultura financiera permitiendo mejorar la toma de decisiones relacionadas en las finanzas personales de la población.

Asimismo, en Garay (2016) se analiza el alfabetismo financiero, utilizando la metodología de componentes principales. La investigación concluye que es importante incorporar la educación financiera en los currículos escolares a fin de reducir las brechas en el alfabetismo financiero de la población. Además, los resultados del estudio indican que los factores culturales inciden significativamente en el alfabetismo financiero al igual que el individualismo, la masculinidad y la evasión de incertidumbre que afectan las decisiones económicofinancieras del individuo. En relación con la masculinidad, se señala que en su comportamiento los valores dominantes son el éxito material y el progreso, lo que supone que los hombres deben ser seguros, agresivos y ambiciosos.

Las aportaciones abordadas como antecedentes ponen en distintos contextos las variables consideradas para la presente investigación estudiando factores como la educación financiera, la regulación financiera por medio de políticas bancarias y estudios de indicadores macroeconómicos, instrumentos financieros y el género. Por lo que, en la presente investigación, se plantea incorporan en un modelo estos factores con el objetivo de identificar los factores que inciden en la cultura financiera de los estudiantes de maestría de la Facultad de Contaduría Pública y Administración de la UANL bajo un enfoque probabilístico con el modelo Probit.

\section{MARCO TEÓRICO}

\subsection{Cultura financiera}

En un orden inicial de ideas la cultura financiera se podría definir como el conjunto de conocimientos que permiten al individuo desarrollar un juicio crítico con respecto a su dinero. La cultura según la UNESCO (1982) puede considerarse como el conjunto de rasgos distintivos, espirituales, materiales, intelectuales, así como afectivos que describen a la población. Esta definición de cultura se plantea desde un punto de vista distinto, considerando rasgos espirituales, materiales y afectivos, adicionales a los conocimientos o rasgos intelectuales. Por otro lado, Ochoa (2009) se define las finanzas como una subdisciplina de la economía relacionada con el estudio de las actividades de inversión, tanto en activos reales como en activos financieros, así como con la administración de éstos. De esta manera es posible considerar a la cultura financiera como el conjunto de rasgos distintivos que caracterizan a alguien y que permiten desarrollar un juicio crítico con respecto a la administración de sus activos.

Con el fin de ampliar el conocimiento del concepto de cultura financiera es necesario realizar un análisis complementario, para ello, se exploran distintas aceptaciones de la cultura financiera elaborados en investigaciones previas realizadas por diversos autores. Por su parte, Varela-Patiño et al. (2016) definen cultura financiera como los conocimientos, 
destrezas y habilidades que permiten entender con claridad conceptos como el ahorro, los ingresos, costos de bienes y servicios, presupuesto personal o el consumo responsable. La cultura financiera entonces puede también considerarse como un conjunto de características propias de un individuo con conocimiento de sus acciones en un entorno económico. Una persona con una buena cultura financiera conoce de conceptos como el ahorro, consumo responsable y presupuesto, los cuales aprovecha para su beneficio, también distingue entre ingreso y crédito, y sabe discernir entre precios de producto $\mathrm{y}$ necesidades básicas como otros bienes de consumo prescindibles.

Por su parte, Amezcua (2014) también define cultura financiera como un conjunto de conocimientos, prácticas, hábitos y costumbres que cada individuo tiene, pero agrega a la definición que estos conocimientos los utiliza el individuo en las diferentes etapas de su vida con el fin de administrar, incrementar y proteger su patrimonio. Por medio de este concepto se consideran factores que pueden ser adquiridos por otros canales distintos a la educación financiera y que pueden ser estimulados también por otros medios como las regulaciones sobre las condiciones financieras del entorno de los individuos, además se integra el elemento del objetivo, como lo es el incrementar y proteger el patrimonio, siendo este un medio para cubrir las necesidades personales más elementales.

Asimismo, la cultura financiera centra el conocimiento o la capacidad de utilizar el conocimiento e incluso la confianza de las personas en las propias acciones financieras (Zait \& Bertea, 2014). De tal manera que el sentido de una dependencia externa de otros para desarrollar la propia cultura financiera, es decir adquirir un conjunto de conocimientos y herramientas útiles por medio de la experiencia de los demás con quienes se tiene relación así sea en el mismo entorno o fuera de él para poder llevar a cabo los objetivos citados anteriormente.

En conclusión, se considera la cultura financiera como el conjunto de rasgos distintivos, de conocimientos y herramientas adquiridos formados por medio de la experiencia propia o adquirida de otros dentro de un entorno económico, y que permiten desarrollar un juicio crítico con respecto a la administración de sus activos, con la finalidad de incrementar y proteger el patrimonio.

\subsection{Género}

En diversos estudios la población se discrimina en grupos, entre ellos se realizan comparaciones por género, por esta razón es razonable realizar la pregunta; ¿El género es un factor incidente en la cultura financiera? El término de género se puede definir como las características de los hombres y las mujeres que están socialmente determinadas, en contraste con aquellos que están biológicamente determinados (UNESCO, 2000), es decir, que estas características son distintas a las sexuales y establecen diferencias entre los individuos desde una perspectiva distinta.

Es irreflexivo considerar el género como un factor natural incidente en la falta o no de cultura financiera, pero es posible considerar al género por las diferencias en las características que este tiene en el comportamiento en cuanto a las finanzas de las personas se refiere, el cual influye en la determinación de la cultura financiera de estas. En 1955 John Money ya definía el rol de género como todo lo que una persona dice y hace para indicar el grado en que es hombre o mujer, (Coleman, 1991) en ese sentido existe una diferencia en las acciones de los individuos y estas definen su identidad de género en una clasificación que como anteriormente se menciona podría ser un factor de diferenciación en la conducta financiera.

Otra visión del rol de género establece una serie de conductas estereotipadas por la cultura, tal y como lo redacta el Instituto Nacional de las Mujeres, (INMUJERES, 2007) se predisponen las acciones que deberían tomar los individuos tomando como base su sexo, y en esta percepción, es 
el sexo el que va a determinar su género y sus acciones, las cuales ya están establecidas con antelación, es entonces importante analizar los estereotipos y criterios relacionados con el género para entender las conductas que afectan la cultura financiera de los individuos.

Es necesario establecer una base para tratar el género como un factor debido a las definiciones diversas presentadas en los párrafos anteriores, se puede aceptar el género como una manifestación de conductas que lo caracterizan y por otro lado, pueden considerarse estereotipos y predisposiciones que minimizan la importancia de un real análisis del comportamiento financiero de los individuos.

El género, se establece entonces, como un factor que identifica una correlación entre las acciones que definen la identidad, las cuales se representan en los hábitos de consumo y comportamiento financiero de ambos géneros, el cual sin intención de establecer un juicio podría incidir de alguna manera a la cultura financiera.

\subsection{Educación financiera}

La educación es el medio por excelencia para adquirir los conocimientos necesarios para desarrollar el juicio crítico para la administración de los bienes y de esa manera proporcionar bienestar. Así como la cultura financiera es la cultura de las finanzas, la educación financiera sería a su vez la educación de las finanzas. La palabra educación quiere decir crianza, enseñanza y doctrina que se da a los niños y a los jóvenes, y las finanzas como se mencionó con anterioridad es la rama de la Economía que se relaciona con el estudio de las actividades de inversión tanto en activos reales como en activos financieros, y con la administración de estos. Por lo que la educación financiera se pudiera definir como la enseñanza de la inversión y administración de activos.

En otra aceptación para la educación financiera, esta es definida como el proceso por medio del cual los individuos mejoran su comprensión de los productos, conceptos y riesgos financieros, además que desarrollan habilidades para ser más conscientes en la toma de decisiones financieras, realizan elecciones informadas y adoptan acciones para mejorar su bienestar (OECD, 2005). La educación financiera en un sentido es el proceso mediante se adquieren los conocimientos y se desarrollan los rasgos intelectuales mencionados en la definición de la cultura financiera. Esto establece, sin la intención de hacer un juicio, una relación directamente proporcional entre la cultura financiera y la educación financiera, donde a medida que se tenga más educación financiera debería de haber más cultura financiera.

Uno de los principales factores para alcanzar el desarrollo humano, social y económico de un país es la educación. Las cambiantes condiciones económicas y sociales han dado a la educación un papel cada vez más central en el éxito de individuos y naciones. (OECD, 2005) Es grande la importancia de la educación para el desarrollo, y en un sentido de finanzas personales la educación financiera permite el desarrollo individual que potencializa el desarrollo económico a un nivel macro.

Domínguez (2013) señala que la educación financiera comprende tres aspectos clave: 1) adquirir conocimiento en materia de finanzas; 2) desarrollar competencias en ese ámbito, y 3) ejercer la responsabilidad financiera, realizando elecciones informadas y con conocimiento de los riesgos asumidos. Los tres aspectos clave mencionados comprenden el proceso del desarrollo de la cultura financiera por medio de la educación, adquirir conocimiento en materia de finanzas, desarrollar su uso y utilizarlas. Una de las principales necesidades para la administración de las finanzas personales es la información, por medio de la educación financiera los individuos pueden tomar decisiones de manera informada dentro del entorno económico al que pertenezcan.

Los programas de estudio del nuevo modelo educativo de educación básica para el periodo 2017-2020 de la Secretaría de 
Educación Pública no contemplan la educación financiera o algo similar dentro de los campos de formación académica y las áreas de desarrollo personal y social (SEP, 2017). Existen opiniones encontradas en cuanto a qué y cómo enseñar sobre finanzas personales a los niños y jóvenes. A menudo se trabaja para evitar una exposición temprana de los niños a temas que se cree son "solo para adultos", pero no se piensa que la comprensión de las finanzas les facilitará en un futuro su toma de decisiones. El problema no reside en enseñar temas de adultos a los niños, sino en transmitirles conocimientos erróneos, o confiar en que otras personas les explicarán los temas financieros (CONDUSEF, 2016).

$\mathrm{El}$ incursionar en la educación financiera y desarrollar habilidades relacionadas a esta durante la educación básica pudiera tener un efecto positivo en las finanzas personales de los futuros usuarios del mercado y de los servicios financieros. La importancia de los programas de educación financiera en los primeros niveles de formación en países latinoamericanos está confirmada también por la participación de estos en el Programa para la Evaluación Internacional de Alumnos (PISA) de la OCDE (García et al., 2013). La evaluación de los conocimientos de educación financiera a nivel global es de gran relevancia por lo que es necesaria su evaluación, las acciones para acercar y mejorar la educación financiera en países como México, y medidas como la inclusión en la prueba PISA confirman su importancia.

Al no existir un programa de estudio relacionado con la educación financiera dentro de los modelos educativos, para poder adquirir los conocimientos y desarrollar las habilidades necesarias en materia financiera es posible optar por la búsqueda de información por cuenta propia mediante el uso de herramientas como el internet o programas gubernamentales. Existen programas de gobierno como la semana de la educación financiera la cual es difundida por distintos canales y otros servicios brindados por la CONDUSEF. El objetivo principal es desarrollar este tipo de conocimiento con un fin práctico, para ayudar a las personas a usar de manera más eficiente sus recursos o conocer y usar los servicios financieros ofertados en el mercado.

En conclusión, la importancia de la educación financiera se encuentra en la adquisición de conocimiento y herramientas para el desarrollo de los rasgos intelectuales necesarios para la cultura financiera, la educación financiera se puede adquirir por medio de programas de educación o por la búsqueda de información por cuenta propia

\subsection{Bancarización}

La bancarización consiste en el acercamiento a realizar estas operaciones financieras por medio de los bancos e instituciones financieras dentro del entorno económico, esta puede definirse también como el nivel de acceso y el grado de utilización de los servicios financieros en general y bancarios en particular (Anastasi, et al., 2010)

La banca es el medio por la cual se llevan a cabo formalmente las inversiones y la administración de los activos dentro del entorno económico. En la medida en que los usuarios utilicen estos servicios el nivel de bancarización aumenta y con ello las opciones para llevar a cabo la administración de estos activos también, por lo que se infiere una correlación directa con la cultura financiera.

Los servicios bancarios cumplen un rol importante en la economía ya que permiten movilizar ahorro y canalizarlo hacia la inversión, logrando a partir de la especialización reducir los costos de información y facilitar las transacciones al tiempo que mediante la diversificación permiten reducir los riesgos y aumentar los retornos. La importancia de este rol de los servicios bancarios se refleja en la evidencia empírica que presenta una correlación positiva entre desarrollo económico y profundidad financiera (Anastasi et al., 2010). La bancarización es útil como un motor de desarrollo toda vez que su 
funcionalidad permite a los usuarios bancarizados orientar de una manera eficiente los recursos, así es posible llegar a más usuarios y fomenta un mejor sistema financiero.

Un elemento importante del sistema financiero en México está formado por los bancos y las empresas que se dedican al ejercicio de la banca quienes constituyen según el organigrama elaborado por la Secretaría de Hacienda y Crédito Público las Instituciones de crédito, de banca múltiple y de banca de desarrollo (Méndez, 2013). Los bancos tienen un papel significativo dentro del sistema financiero mexicano, por medio de ellos es posible configurar instrumentos para los usuarios y conseguir el desarrollo económico del que se habla en los párrafos anteriores.

La bancarización como señala Morales \& Yañez (2006) implica mucho más que el acceso al crédito, también se refiere al establecimiento de relaciones estables y amplias entre las instituciones financieras y sus usuarios, respecto de un conjunto de servicios financieros disponibles por las instituciones financieras hacia los usuarios y la capacidad que estos tienen de poder utilizarlos dentro del sistema financiero. En principio pueden distinguirse tres tipos básicos de servicios bancarios, 1) medios de pago o servicios transaccionales, 2) ahorro y 3) crédito.

Con el propósito de hacer más fácil las operaciones comerciales y financieras, los bancos han creado instrumentos de pago, como, por ejemplo, las tarjetas de crédito o débito y los cheques. Estos instrumentos de pago permiten pagar cantidades exactas sin la necesidad de llevar billetes. Asimismo, se han desarrollado otras formas de pago, como las transferencias por Internet que logran hacer pagos sin tener que ir a un banco (Banxico, 2018). El uso de los instrumentos de pago creados por los bancos constituye un medio de acercamiento a la bancarización. En la actualidad se han desarrollado medios electrónicos que facilitan las transacciones en el sistema financiero.

Otra manera de acercarse a la bancarización y desarrollar la cultura financiera se logra utilizando los instrumentos crediticios que la banca ofrece, como las tarjetas de crédito y otros créditos en diferentes condiciones. Con el correcto manejo de la tarjeta de crédito, no sólo comenzarás una reputación crediticia favorable, sino que te servirá para formarte el hábito de utilizar el crédito de manera responsable (CONDUSEF, 2014). La importancia del uso del crédito dentro del sistema financiero reside en crear un historial y facilitar de esta manera el acercamiento a créditos para la adquisición de distintos bienes como automóviles y bienes inmuebles por medio de créditos hipotecarios.

Guardar tu dinero en una institución autorizada por la Comisión Nacional Bancaria y de Valores (CNBV) y por la CONDUSEF, proporciona seguridad de que el dinero se encuentre protegido y evitar futuras afectaciones (CONDUSEF, 2014). Una de las ventajas de la bancarización es la seguridad que se obtiene al tener los activos almacenados en instituciones financieras y las garantías que otorgan organismos como la CONDUSEF con respecto al manejo de estos recursos, además de los rendimientos que se tienen por inversiones y otros instrumentos financieros.

La bancarización en México representa un problema de inclusión financiera para gran parte de la población, existe un número considerable de adultos que continúan fuera de la bancarización debido a que no utilizan los medios de pago, ahorro y crédito que ofrecen los bancos e instituciones financieras en el país. En conclusión, la bancarización es el nivel de acceso a la utilización de los servicios financieros como los medios de pago, ahorro y crédito que se realizan por medio de los bancos e instituciones financieras, y la relación con estas instituciones financieras y los bancos para el desarrollo de la cultura financiera.

\subsection{Regulación financiera}

La regulación financiera se entiende como el 
marco legal que regula las operaciones financieras que se mencionadas anteriormente, se refiere a la acción de regular las finanzas, la palabra regular quiere decir ajustar el funcionamiento de un sistema a determinados fines estableciendo las reglas o normas a que debe ajustarse. Por lo que la regulación financiera se puede definir como la acción de establecer reglas o normas para ajustar el funcionamiento de la inversión y administración de los activos. Las entidades reguladoras y supervisoras son las encargadas de llevar a cabo la regulación financiera.

En México, el sistema financiero está integrado por organizaciones gubernamentales y no gubernamentales, para establecer mediante leyes, reglamentos, circulares, etc. el control de las operaciones entre las instituciones financieras del país, su objetivo es ordenar la conducta y operación de las instituciones bancarias, bursátiles y auxiliares de crédito a través de órganos de vigilancia, supervisión y control (Sánchez, 2010). El sistema de entidades reguladoras y supervisoras en México consta de instituciones del estado y órganos descentralizados encargados de regular la relación de las instituciones y los usuarios financieros, así como el proceder y limitaciones de ambas partes decretadas por medio de las leyes promulgadas en el país.

Actualmente, la regulación y la supervisión del sistema financiero descansan principalmente en cinco entidades gubernamentales: la Secretaría de Hacienda y Crédito Público (SHCP), el Banco de México (Banxico), la Comisión Nacional Bancaria y de Valores (CNBV), el Instituto para la Protección al Ahorro Bancario (IPAB) y la Comisión Nacional para la Defensa de los Usuarios de Servicios Financieros (CONDUSEF). Las entidades gubernamentales tienen la misión de llevar a cumplimiento los mandatos legales en materia de fiscalización, bancarización y finanzas dentro del sistema de financiero por medio de las herramientas y capacidades que la ley les otorga (BBVA, 2000).

Existen leyes promulgadas en el país que conforman el marco legal, además de las circulares emitidas por el Banco de México y medidas de transparencia como el indicador de Costo Anual Total (CAT) y Gasto Anual Total (GAT). En conclusión, la regulación financiera es el conjunto de leyes que establecen las reglas o normas para ajustar el funcionamiento de la inversión y administración de los activos por medio de las entidades reguladoras apegadas al marco legal dentro del sistema financiero del país.

\section{MÉTODO}

Considerando los estudios previos se plantea la siguiente hipótesis general: Los factores género, educación financiera, bancarización y regulación financiera inciden en la cultura financiera de los estudiantes de maestría de la Facultad de Contaduría Pública y Administración de la Universidad Autónoma de Nuevo León.

Para comprobar la hipótesis se plantea el siguiente diseño de investigación. El estudio es de tipo cuantitativo, toda vez que plantea un problema sujeto de estudio delimitado y concreto. Además, con las características del enfoque cuantitativo destaca que el problema central de la investigación ha sido abordado por diferentes autores particularmente en los temas de género, educación financiera, bancarización, regulación y cultura financieras. Asimismo, la presente investigación es cuantitativa, considerando que durante el desarrollo de la misma se recolectan datos por medio del instrumento de medición, que nos permite comprobar la incidencia de las variables estudiadas con la cultura financiera por medio de medición numérica y el análisis estadístico.

La investigación es cuantitativa no experimental, ya que los estudios se realizan sin la manipulación de variables y está diseñada conforme a aspectos exploratorios, descriptivos y correlacionales. Durante el desarrollo de la investigación se aplica la técnica de investigación cuantitativa denominada transaccional, en esta técnica se realiza la recolección de datos en un único momento.

La unidad de análisis sujeta de estudio 
del presente trabajo de investigación, es decir, la población, se integra por 227 estudiantes matriculados en las distintas maestrías del posgrado de la Facultad de Contaduría Pública y Administración (FACPyA) durante el periodo escolar eneroabril del año 2018.

Para realizar el estudio se determinó un tamaño de muestra de 55 estudiantes por medio de un muestreo aleatorio simple con una precisión de $5 \%$ y nivel de confianza del 95\% como sigue:

$$
n=\frac{\left(227 * 1.96^{2}\right) *(0.05 * 0.95)}{0.05^{2}(227-1)+1.96^{2}(0.05 * 0.95)}=55
$$

Se determinó utilizar como técnica para la recolección de datos una encuesta estructurada en forma personal, la cual se aplica a los estudiantes de Maestría en la FACPyA durante el periodo escolar eneroabril del año 2018 de manera presencial en las aulas por medio de un formulario impreso y reproducido.

El instrumento de medición (cuestionario) que se utiliza en la presente investigación para recolección de los datos, los cuales son necesarios para realizar los análisis se constituye en dos secciones; la primer sección consta de preguntas de tipo general en las que se destacan las correspondientes a la edad, género, maestría cursando, ingresos y nivel de cultura financiera, las cuales se utilizan para delimitar y analizar las características de los encuestados y relacionarlas con los factores que inciden en la cultura financiera y la segunda sección del instrumento de medición corresponde a preguntas con respuestas en escala Likert, en la que se pretende medir la percepción que tienen los encuestados con respecto a las variables que tienen una incidencia en la cultura financiera de los estudiantes de maestría de la FACPyA, como son el género, la educación financiera, la bancarización y las regulación financiera.

Con la información generada por los medios anteriormente mencionados se realizan análisis de confiabilidad y factorial confirmatorio utilizando el software SPSS, por último, se efectúa una prueba de coeficiente por el modelo Probit utilizando el paquete Eviews.

\section{RESULTADOS}

Primero, se realiza una prueba de confiabilidad de la encuesta la cual se determina mediante el coeficiente alfa de Cronbach. Se utiliza este instrumento para efectos de verificar en una medida la confiabilidad de los ítems del instrumento de medición aplicado, para esto se toma el siguiente criterio de interpretación de los resultados donde; $>.9$ es excelente, $>.8$ es bueno, $>.7$ es aceptable, $>.6$ es cuestionable $y>.5$ es pobre. Tomando en cuenta el criterio citado con anterioridad y con un alfa de Cronbach determinado con la herramienta SPSS de .714 se considera que la confiabilidad del cuestionario es aceptable.

Las preguntas del apartado 1 proporcionan los resultados concernientes a las características de los encuestados, de estas se destacan principalmente el género, edad, maestría en curso, ingresos y nivel de cultura de financiera.

- Con respecto al generó, se detalla que del total de los encuestados el $54.5 \%$ son hombres y el $45.5 \%$ son mujeres, cabe señalar que la encuesta se realizó con los alumnos presentes en las aulas durante los días aleatoriamente seleccionados.

- Las edades de los encuestados se concentran entre los 22 y 27 años, con un número mayor de encuestados con 25 años con un $80 \%$ del total de los encuestados.

- Del total de los encuestados el $40.00 \%$ tienen ingresos mensuales de 15,001 a 25,000 mientras que el $30.91 \%$ dice tener ingresos por más de 25,000 pesos al mes.

- De la participación de los encuestados por maestría, en esta se destaca con un 43.64\% de los encuestados la Maestría en Contaduría con orientación en Finanzas y sin participación la Maestría en Administración de empresas con orientación en Gestión Pública, Maestría en Contaduría con orientación en Auditoria y Master of Business 
Administration con orientación en Mercadotecnia Global.

- Por último, se hace un análisis del nivel de cultura financiera que consideran tener los alumnos de nivel maestría de la FACPyA, del total de los encuestados el $50.91 \%$ tiene un suficiente nivel de cultura financiera y un $45.45 \%$ tiene algo de cultura financiera.

A continuación, se señalan los resultados del apartado 2 de la encuesta que describen la percepción con respecto a la incidencia que tienen las variables independientes en la cultura financiera de los estudiantes de maestría:

- Del total de los encuestados el $60.00 \%$ está totalmente en desacuerdo con que el género incide en la cultura financiera de los estudiantes de maestría de la FACPyA.

- Del total de los encuestados el $40.00 \%$ está en acuerdo con la incidencia de la alfabetización financiera en la cultura financiera de los estudiantes de maestría, además el $47.27 \%$ está en acuerdo con la afirmación que refiere que los estudiantes de maestría tienen una mejor cultura financiera y el $47.27 \%$ está totalmente de acuerdo con que buscar educación financiera por cuenta propia es útil para tener una mejor cultura financiera. En otro resultado el $41.82 \%$ está en acuerdo y $34.55 \%$ totalmente de acuerdo con la necesidad de la educación financiera desde la educación básica para tener una mejor cultura financiera.

- En los resultados correspondientes a la variable de bancarización se destaca que el $36.36 \%$ está de acuerdo y el $20.00 \%$ totalmente de acuerdo con que el uso de la banca es importante para tener una buena cultura financiera. El 32.73\% está en desacuerdo y el $29.09 \%$ totalmente en desacuerdo que las tarjetas de crédito son utilizadas por personas con buena cultura financiera. Además, el $41.82 \%$ no está ni de acuerdo ni en desacuerdo que el ahorrar en bancos sea una actividad de las personas con buena cultura financiera.

- En estos resultados se destaca que el $40.00 \%$ está de acuerdo que las autoridades reguladoras en materia financiera son importantes para tener una mejor cultura financiera y también que el $45.45 \%$ no está ni de acuerdo ni en desacuerdo con la importancia de la afectación de las leyes financieras en la cultura financiera de los estudiantes de maestría de la FACPyA.

Para realizar el análisis factorial confirmatorio (AFC) se hace una clasificación de las preguntas contenidas en la encuesta en constructos representados por las variables de género, educación financiera, bancarización y regulación financiera (véase Tabla 1).

Tabla 1. Construcción de los constructos del estudio.

\begin{tabular}{ll}
\hline Constructo & Ítems $^{(\mathbf{1})}$ \\
\hline Género & $\mathrm{P} 1, \mathrm{P} 2, \mathrm{P} 3$ \\
Educación financiera & $\mathrm{P} 4, \mathrm{P} 5, \mathrm{P} 6, \mathrm{P} 7$ \\
Bancarización & $\mathrm{P} 8, \mathrm{P} 9, \mathrm{P} 10$ \\
Regulación financiera & $\mathrm{P} 11, \mathrm{P} 12, \mathrm{P} 13$ \\
\hline
\end{tabular}

\footnotetext{
(1) Preguntas del cuestionario
}

Fuente: Elaboración propia 
En la Tabla 2 se presentan los resultados de la reducción de factores para la comprobación de los constructos por medio de una prueba de Matriz de componente rotado por el método de extracción de análisis de componentes principales con un método de rotación Varimax con normalización de Kaiser ajustado a cuatro constructos.

Tabla 2. Matriz de componente rotado.

\begin{tabular}{ccccc}
\hline Pregunta & Factor 1 & Factor 2 & Factor 3 & Factor 4 \\
\hline P2 & .934 & -.040 & -.036 & .061 \\
P3 & .909 & -.030 & -.104 & .059 \\
P1 & .804 & -.115 & -.105 & -.221 \\
P8 & -.125 & .755 & .117 & .149 \\
P10 & -.087 & .714 & -.039 & -.100 \\
P12 & .001 & .690 & .186 & -.190 \\
P9 & .038 & .671 & .084 & .121 \\
P4 & -.040 & .002 & .845 & -.032 \\
P11 & -.277 & .270 & .745 & .035 \\
P5 & -.243 & .033 & .686 & .262 \\
P13 & .230 & .108 & .384 & -.036 \\
P7 & .006 & .172 & -.113 & .864 \\
P6 & .064 & -.197 & .261 & .681 \\
\hline
\end{tabular}

Método de extracción: análisis de componentes principales.

Método de rotación: Varimax con normalización Kaiser.

La rotación ha convergido en 5 iteraciones

Fuente: Elaboración propia utilizando el software SPSS.

De los resultados presentados en la Tabla 2 destaca una rotación convergente en cinco iteraciones por lo que se realizan ajustes de los items utilizados para la prueba descartando las preguntas P7, P11, P12 y P13 de la aplicación del instrumento medición. Después de los ajustes realizados se realiza nuevamente la construcción estadística de los constructos por medio de Matriz de componente rotado converge en tres iteraciones (véase Tabla 3).

Tabla 3. Matriz de componente rotado ajustado.

\begin{tabular}{cccc}
\hline Pregunta & Factor 1 & Factor 2 & Factor 3 \\
\hline P2i & .930 & .061 & .040 \\
P3i & .911 & .031 & .099 \\
P1i & .820 & .086 & .160 \\
P9 & -.086 & .764 & .174 \\
P8 & .138 & .761 & .046 \\
P10 & .117 & .723 & -.200 \\
P4 & .011 & .089 & .808 \\
P5 & .195 & .171 & .799
\end{tabular}


P6

.075

$-.187$

.501

Notas:

Método de extracción: análisis de componentes principales.

Método de rotación: Varimax con normalización Kaiser.

La rotación ha convergido en 5 iteraciones

Fuente: SPSS Statistics

A continuación, se detallan los constructos resultantes después de los ajustes realizados para la construcción estadística para el AFC, se destaca la eliminación de la variable latente de Regulación financiera y la pregunta 7 del instrumento de medición que explica la correlación de la variable de Educación financiera con la Cultura financiera de los estudiantes de maestría de la FACPyA.

Tabla 4. Constructos de estudio ajustados.

\begin{tabular}{ll}
\hline Constructo & Ítems $^{(\mathbf{1})}$ \\
\hline Género & $\mathrm{P} 1, \mathrm{P} 2, \mathrm{P} 3$ \\
Educación financiera & $\mathrm{P} 4, \mathrm{P} 5, \mathrm{P} 6$ \\
Bancarización & $\mathrm{P} 8, \mathrm{P} 9, \mathrm{P} 10$ \\
\hline
\end{tabular}

${ }^{(1)}$ Preguntas del cuestionario

Fuente: Elaboración propia

Posteriormente, en virtud de que la variable dependiente está en una escala de Likert, se estima un modelo de Probit ordenado con corrección de White para considerar la posible presencia de Heterocedasticidad la cual es común en este tipo de modelos. El modelo se estima utilizando los factores obtenidos con el análisis factorial de la que resultaron tres principales componentes: el género, bancarización y educación financiera, los resultados se muestran en la Tabla 5

Tabla 5. Resultados del modelo Probit.

\begin{tabular}{lcccc}
\hline Variable & Coeficiente & Error Std. & z-Estadística & Prob. \\
\hline Género & 0.063565 & 0.167031 & 0.380555 & 0.7035 \\
Bancarización & -0.303545 & 0.197085 & -1.540175 & 0.1235 \\
Educación Financiera & 0.261391 & 0.150926 & 1.731909 & 0.0833 \\
\hline
\end{tabular}

Fuente: Elaboración propia utilizando el software Eviews.

Para revisar la bondad de ajuste se

es correcto siendo mejor incrementándose este porcentaje cuando la respuesta del encuestado es 3 "Suficiente", véase Tabla 6. observa que en el $58 \%$ de los casos el modelo

Tabla 6. Bondad de ajuste del instrumento de medición (cuestionario).

\begin{tabular}{cccccc}
\hline Valor & Observado & Correcto & Incorrecto & \% Correcto & \% Incorrecto \\
\hline 1 & 1 & 0 & 1 & 0.000 & 100.000 \\
2 & 25 & 13 & 12 & 52.000 & 48.000 \\
3 & 28 & 19 & 9 & 67.857 & 32.143 \\
4 & 1 & 0 & 1 & 0.000 & 100.000 \\
\hline
\end{tabular}




\begin{tabular}{llllll} 
Total & 55 & 32 & 23 & 58.182 & 41.818 \\
\hline
\end{tabular}

Fuente: Elaboración propia utilizando el software Eviews.

Considerando los efectos marginales del modelo Probit estimado con los valores promedio en la Tabla 7 se observa que hay un $43 \%$ de probabilidad de que el encuestado tenga cultura financiera.

Tabla 7. Probabilidad estimada del modelo Probit ordenado con valores promedio.

\begin{tabular}{ccc}
\hline Incremental & Probabilidad & Categoría Likert \\
\hline $9 \%$ & $9 \%$ & 1 \\
$48 \%$ & $39 \%$ & 2 \\
$91 \%$ & $43 \%{ }^{(1)}$ & $3^{(2)}$ \\
$100 \%$ & $9 \%$ & 4 \\
\hline (1) Máxima probabilidad & & \\
(2) Pronóstico & &
\end{tabular}

\section{CONCLUSIONES}

De los resultados del estudio se puede señalar que la hipótesis planteada se rechaza parcialmente, ya que de los cuatro factores analizados sólo en uno de ellos se encontró evidencia de que incidiera en la cultura financiera de los estudiantes. Con ello, se derivan las siguientes conclusiones.

En primera instancia, considerando el resultado obtenido de la reducción de factores por medio del análisis factorial confirmatorio, no se encuentra evidencia que la regulación financiera incide en la cultura financiera de los estudiantes de maestría de FACPyA. En razón a las respuestas, los encuestados consideran que las autoridades reguladoras y las leyes no tienen una afectación en el nivel de cultura financiera. De tal manera que podemos inferir que los cambios en la regulación no tienen necesariamente una afectación directa en la cultura financiera, pero considerando que ésta pudiera incidir en los otros factores es posible que el efecto de esta variable pueda darse de manera indirecta sobre la cultura financiera de los estudiantes de maestría.

En segundo lugar, derivado del análisis del instrumento de medición los estudiantes declaran que hay poco efecto del factor género y bancarización en la falta de cultura financiera de los estudiantes de Maestría de la FACPyA. Por lo que, no se encontró evidencia estadística con el resultado del modelo Probit. Es importante hacer mención que, en el análisis de reducción de factores, estas variables de género y bancarización no fueron descartadas de las variables latentes.

Finalmente, con el resultado de la prueba de Probit se concluye una relación de incidencia de la educación financiera con la variable dependiente. Por medio del instrumento de medición, los encuestados con sus respuestas indican que existe una relación positiva de la educación financiera con la cultura financiera de los estudiantes de maestría, es decir, a medida que se tenga más educación financiera se tendrá más cultura financiera.

En Amezcua (2014) se destaca la importancia de la educación financiera y como ésta se relaciona con la cultura financiera con respecto a los hábitos que tienen los usuarios del entorno económico y financiero en el país. Los bajos niveles de cultura financiera que tiene la población mexicana se deben en gran parte a la falta de educación financiera. En la revisión de estudios previos se destaca una escasa participación de los sectores sociales de los productos y servicios financieros, el desconocimiento de los derechos y 
obligaciones frente a las instituciones financieras, así como la falta de planeación financiera.

Considerando lo anterior, podemos mencionar que la cultura financiera se ve reflejada en elementos del conocimiento y la planeación financiera, elementos que se fomentan por medio de la educación financiera. En este sentido, como señala Acevedo (2014) las finanzas personales pueden mejorar con el conocimiento y aplicación de presupuestos, flujos de caja, manejo de tasas de interés y conocimiento de productos de la banca comercial del país tanto en captación como financiación, con todas estas bases es sencillo tomar decisiones correctas basándose en información real. Estos elementos tienen influencia en la educación financiera, y como se establece en las conclusiones del modelo Probit, la educación financiera tiene una relación positiva con la cultura financiera, dicho de otra forma, a mayor conocimiento de aplicación de presupuestos, flujos de caja, manejo de tasas de interés y productos de la banca comercial los niveles de cultura financiera son mayores.

En conclusión, la educación financiera es una variable que tiene un impacto importante en la cultura financiera de los estudiantes de maestría. Mediante la adquisición de conocimiento y comprensión en materia de finanzas, el desarrollo de competencias en ese ámbito y ejercer la responsabilidad financiera se puede desarrollar esta educación financiera la cual otorga un conocimiento del ahorro, consumo responsable y presupuesto, conceptos conocidos por aquello que cuentan con una buena cultura financiera. 


\section{REFERENCIAS}

Acevedo, L.M. (2014). ¿Cómo mejorar las finanzas personales por medio de la educación financiera en Colombia? Tesis para obtener el grado de especialización en formulación y evaluación de proyectos públicos y privados. Medellin: Universidad de Medellín.

Amezcua, E.L., Arroyo, M.G. \& (2014). Docencia de las aulas a la investigación. Ciencia Administrativa, 1, pp 21-30.

Anastasi, A., Blanco, E., Elosegui, P. \& Sangiácomo, M. (2010). La bancarización y los determinantes de la disponibilidad de servicios bancarios en Argentina. BCRA Ensayos Económicos, 60, pp 137-209.

Banxico (2018). Sistemas de pago. Ciudad de México: Banco de México. Obtenido de http://www.anterior.banxico.org.mx/divulgacion/sistemas-de-pago/sistemas-pago.html

Coleman, E. (1991). John Money: A Tribute. New York: The Haworth Press.

CONDUSEF (2013). Una cronología sobre las acciones en materia financiera. Proteja su Dinero, 162 , pp. 10-19.

CONDUSEF (2014). ¿Por qué es relevante en tu vida? Proteja su Dinero, 175, pp 14-23.

CONDUSEF. (2016). Más que una tendencia. Proteja su dinero, 194, pp. 16-18.

Domínguez, J.M. (2013). Educación financiera para los jóvenes; Una visión introductoria, Documento de trabajo del Instituto Universitario de Análisis Económico y Social, 5, pp. $1-41$.

Garay, G. (2016). Índice de alfabetismo financiero, la cultura y la educación financiera. Revista Perspectivas, 19(37), pp. 23-40.

García, N., Grifoni, A., López, J.C., \& Mejía, D.M. (2013). La educación financiera en América Latina y el Caribe Situación actual y perspectivas. Caracas: Corporación Andina de Fomento, Banco de Desarrollo de América Latina.

BBVA (2000). La regulación y la supervisión bancarias. Ciudad de México: Grupo Financiero BBVA Bancomer.

INMUJERES. (2007). Boletín estadístico: El impacto de los estereotipos y los roles de género en México. Ciudad de México: Instituto Nacional de las Mujeres.

Méndez, J. S. (2013). Problemas económicos de México y sustentabilidad. Ciudad de México: Mc Graw Hill.

Morales, L., \& Yañez, Á. (2006). La bancarización en Chile, concepto y medición. Serie Técnica de Estudios de la Superintendencia de Bancos e Instituciones Financieras, 7, pp 1-33.

Ochoa, G.A. (2009). Administración financiera. Ciudad de México: Mc Graw Hill.

OECD (2005). OECD Annual Report 2005. Paris: Organization for Economic Co-Operation and Development.

Polaina, M.F., Suaza, C., Arévalo, N., \& Gonzalez, D. (2016). La cultura financiera como el nuevo motor para el desarrollo económico en Latinoamérica. Tesis para obtener el grado de especialización en negocios y finanzas internacionales. Bogotá: Universidad EAN.

Sánchez, F. (2010). El sistema financiero de México. Revista UPIICSA, 52, pp 23-35.

SEP (2017). Aprendizajes clave para la educación integral. Ciudad de México: Secretaría de Educación Pública.

UNESCO (1982). Declaración de México sobre las políticas culturales. Conferencia mundial sobre las políticas culturales. Ciudad de México: Organización de las Naciones Unidas para la Educación, la Ciencia y la Tecnología.

UNESCO (2000). Gender sensitivity. Zambia: Organización de las Naciones Unidas para la Educación, la Ciencia y la Tecnología.

Varela-Patiño, C.M., Martínez-Clapé, M. \& Parada-Ulloa, A. (2016). El método de transversalidad financiera empresarial para la formación de la cultura económica y financiera. Santiago, 140, pp. 280-291. 
Zait, A., \& Bertea, P.E. (2014). Financial Literacy - Conceptual Definition and Proposed Approach for a Measurement Instrument. The Journal of Accounting and Management, 4(3), pp. 3742 